\title{
Trying to Prevent the Clogged Drain: Optimizing the Yield and Function of Portal Vein-Infused Islets
}

\author{
A. N. Balamurugan - Timothy L. Pruett
}

Published online: 7 May 2013

(c) Springer Science+Business Media New York 2013

Total pancreatectomy with islet auto-transplantation (IAT) is a major surgical procedure during which the pancreas gland is resected, and pancreatic islets are isolated then infused into the patient's portal vein. To avoid complications it is desirable to minimize the total volume of tissue transplanted intraportally [1]. Embolization via intra portal (IP) infusion of islets in auto and allo-transplantation can be associated with portal hypertension (PH), hepatic infarction, elevation of liver enzyme levels (transaminitis), disseminated intravascular coagulation, and portal venous thrombosis (PVT) [2-5]. Two recent articles in Digestive Diseases and Sciences, one in this issue, seek to address issues associated with isolation and intraportal infusion of islets. Khan et al. [6] report the use of preoperative MRI with contrast to predict of extent of pancreatic fibrosis and subsequent islet yield. This is a very useful report, but would benefit from discussion of whether any modification of the islet digestion technique would improve yield. We need a reproducible method for estimation of total potential islet mass/number within the pancreas that correlates with the difficulty of islet isolation. The article by Khan is a step toward a pre-isolation estimate of islet availability by use of MRI, but stating that the more diseased gland has fewer recoverable islets still leaves many questions relating to process optimization. However, as noted below, optimization of the islet isolation technique has yet to be defined for the fibrotic pancreas.

Isolating islets from the pancreas of a patient with chronic pancreatitis (CP) poses multiple challenges. During islet isolation, collagenase digestion separates islets from

\footnotetext{
A. N. Balamurugan · T. L. Pruett $(\bowtie)$

Department of Surgery, Schulze Diabetes Institute, University of Minnesota, Minneapolis, MN, USA

e-mail: tlpruett@umn.edu
}

exocrine cells; total tissue volume collected varies depending on donor characteristics, pancreatic fibrosis, the pathological status of the exocrine cells, and the efficiency of islet isolation [7]. The typical autologous islet transplant product often contains as much exocrine as endocrine tissue. The technical difficulty of islet isolation is daunting. Enzyme source and manufacturer, time, pressure, and temperature, in addition to the host factors listed above, affect islet yield from the diseased pancreas. Aggressive purification steps can significantly reduce islet yields. Transplantation of unpurified islet preparations is, therefore, still preferred during IAT. Portal vein flow complications/thrombosis or significant liver dysfunction are virtually unheard of for small-volume islet preparations ( $<10 \mathrm{cc}$ volume). When pellet volumes exceed $20 \mathrm{cc}$, however, our practice is to perform further islet purification to reduce the risk of complications. Even with improvements in large-scale purification techniques using the COBE 2991 cell processor, purification unavoidably reduces yield [8].

The article by Desai et al. [9] in this issue reports the relationship between liver histology and the development of transaminitis after autoislet transplantation. Among their 26 autologous islet transplant cases, three had PVT. It was concluded that preexisting liver pathology was a factor contributing to the increase in liver enzymes but did not correlate with development of PVT. Generally, hepatocyte injury is multifactorial; likely causes include hypoxia, thrombosis, inflammation from infused cellular debris, a possible direct toxic effect of the isolation process, and host factors. Desai study [9] supports the notion that liver susceptibility to islet infusion and alteration of portal blood flow is variable in patients undergoing pancreatectomy and autoislet transplantation. The increase in liver enzymes in this series was relatively modest, returning to normal in all 
patients, including those with PV occlusion/thrombosis, similar to the conclusions reported by Barshes et al. [10] for a series of 26 allo-transplanted subjects. They concluded that post-transplant transaminitis was not indicative of acute rejection or serious procedure-related complications, for example PVT or intrahepatic hematoma.

The 6th annual report of the Collaborative Islet Transplant Registry (CITR) showed that partial branch-vein occlusions were identified in $2.2 \%$ of transplanted patients [11]. A previous report from an international multicenter trial suggested that covert, underlying thrombophilic tendencies (protein $\mathrm{C}, \mathrm{S}$, antithrombin III, factor v Leiden deficiencies, or presence of antiphospholipid antibodies) may potentially exacerbate the risk of portal thrombosis after islet transplantation [12]. In a large, single-center prospective series of 268 consecutive IP islet allo-transplants conducted over 11 years, the Edmonton group recently reported their incidence of portal venous thrombosis [13]. Kawahara et al. analyzed the contributory factors leading to partial thrombosis, including standard liver volume (SLV), packed cell volume, and acute portal pressure rise after islet transplantation, assuming that a smaller liver volume would be likely to have less capacity for embolized IP tissue. They also further investigated whether partial portal thrombosis was associated with detrimental loss of islet functional reserve. The incidence of portal thrombosis was low (3.2\%); despite partial portal thrombosis there was no detectable loss of islet graft function as measured by the SUITO index or insulin requirements [13, 14]. Furthermore, Bucher et al. [15] reported no association between portal thrombosis and increased IP pressure in islet autograft and allogeneic transplants when they limited the tissue volume infused to a mean of $13 \mathrm{cc}$ [15].

In our institution, we examined a large cohort of TPIAT patients $(n=233)$ to determine major risk factors for elevated portal pressure during islet infusion. Univariate and multivariate analysis of numerous patient demographics and islet product characteristics indicated that total tissue volume per kg body weight infused intraportally is the best predictor of change in portal pressure $(\Delta \mathrm{PP})$ $\left(p<0.0001 ; R^{2}=0.566\right)$. Baseline incidence of bleeding requiring post-operative intervention and portal venous thrombosis was 7.73 and $3.43 \%$, respectively. Both TV $/ \mathrm{kg}$ and $\Delta \mathrm{PP}$ are associated with greater incidence of complications, although $\triangle \mathrm{PP}$ seems to be more clinically relevant. Receiver operating characteristic (ROC) analysis indicated that only the model for $\triangle \mathrm{PP}$ and thrombosis was relevant, with a suggested cut-point of $27 \mathrm{cmH}_{2} \mathrm{O}(\mathrm{AUC}=0.759$ ). On the basis of this analysis, we recommend $0.25 \mathrm{cc} / \mathrm{kg}$ for $\mathrm{TV}$ and $25 \mathrm{cmH}_{2} \mathrm{O}$ for $\Delta \mathrm{PP}$. These thresholds, the predictive model, and relative risk statistics are useful information for islet processing after isolation and clinical decision-making.
Although the risks of bleeding and PVT have been reported [2-5], these complications may be entirely preventable by use of a combination of systemic heparin and limited pancreatic tissue volume. Complete thrombosis of the entire portal venous system is the most feared complication of IP islet transplantation. In many reported studies, portal venous pressure was significantly correlated with packed cell volume. However, complete thrombosis, with the consequences of chronic portal hypertension, is now extremely rare.

Although intrahepatic infusion of unpurified pancreatic islets affects portal vein pressure and hepatic biochemical profile, these effects are transient and do not compromise the safety of the procedure. Infusion of unpurified islets should be performed with great caution, although increased understanding of the pathophysiology of complications combined with technical improvements have improved the safety of the procedure substantially, as reported in the two highlighted articles.

Acknowledgments We thank Joshua Wilhelm, Mukesh Tiwari and Gopalakrishnan Loganathan for their contributions.

\section{References}

1. Sutherland DE, Radosevich DM, Bellin MD, et al. Total pancreatectomy and islet autotransplantation for chronic pancreatitis. $J$ Am Coll Surg. 2012;214:409-424.

2. Casey JJ, Lakey JR, Ryan EA, et al. Portal venous pressure changes after sequential clinical islet transplantation. Transplantation. 2002;74:913-915.

3. Mehigan DG, Bell WR, Zuidema GD, et al. Disseminated intravascular coagulation and portal hypertension following pancreatic islet transplantation. Ann Surg. 1980;191:287.

4. Walsh TJ, Eggleston JC, Cameron JL. Portal hypertension, hepatic infarction, and liver failure complicating pancreatic islet autotransplantation. Surgery. 1982;91:485-487.

5. Shapiro AM, Lakey JR, Rajotte RV, et al. Portal vein thrombosis after transplantation of partially purified pancreatic islets in a combined human liver/islet allograft. Transplantation. 1995;59:1060-1063.

6. Khan K, Desai CS, Kalb B, et al. MRI prediction of islet yield for autologous transplantation after total pancreatectomy for chronic pancreatitis. Dig Dis Sci. 2013;58:1116-1124.

7. Balamurugan AN, Loganathan G, Bellin MD, et al. A new enzyme mixture to increase the yield and transplant rate of autologous and allogeneic human islet products. Transplantation. 2012;93:693-702.

8. Anazawa T, Matsumoto S, Yonekawa Y, et al. Prediction of pancreatic tissue densities by an analytical test gradient system before purification maximizes human islet recovery for islet autotransplantation/allotransplantation. Transplantation. 2011;91: 508-514.

9. Desai CS, Khan KM, Megawa FB, et al. Influence of liver histopathology on transaminitis following total pancreatectomy and autologous islet transplantation. Dig Dis Sci. (Epub ahead of print). doi:10.1007/s10620-012-2264-7.

10. Barshes NR, Lee TC, Goodpastor SE, et al. Transaminitis after pancreatic islet transplantation. J Am Coll Surg. 2005;200:f353f361. 
11. Report CITR. CITR Annual Report. 2009 Available from: https://web.emmes.com/study/isl/reports/reports.htm.

12. Brennan DC, Shannon MB, Koch MJ, Polonsky KS, Desai N, Shapiro J. Portal vein thrombosis complicating islet transplantation in a recipient with the Factor V Leiden mutation. Transplantation. 2004;78:172-173.

13. Kawahara T, Kin T, Kashkoush S, et al. Portal vein thrombosis is a potentially preventable complication in clinical islet transplantation. Am J Transplant. 2011;11:2700-2707.
14. Villiger P, Ryan EA, Owen R, et al. Prevention of bleeding after islet transplantation: lessons learned from a multivariate analysis of 132 cases at a single institution. Am J Transplant. 2005;5: 2992-2998.

15. Bucher P, Mathe Z, Bosco D, et al. Morbidity associated with intraportal islet transplantation. Transplant Proc. 2004;36: 1119-1120. 